\title{
On two new combined 3-Fibonacci sequences. Part 2
}

\author{
Krassimir T. Atanassov \\ Department of Bioinformatics and Mathematical Modelling \\ IBPhBME - Bulgarian Academy of Sciences, Sofia-1113, Bulgaria \\ and \\ Intelligent Systems Laboratory \\ Prof. Asen Zlatarov University, Burgas-8010, Bulgaria \\ e-mail: krat@bas.bg
}

\begin{abstract}
In continuation of the results in [1], two new combined 3-Fibonacci sequences are introduced. Explicit formulae for their $n$-th members are given. In comparison to the combined 3-Fibonacci sequences proposed in [1], which are functions of four initial constants, the new sequences proposed here depend only on three initial constants.
\end{abstract}

Keywords: Fibonacci sequence, 3-Fibonacci sequence.

2010 Mathematics Subject Classification: 11B39.

\section{Introduction}

In [1], two new combined 3-Fibonacci sequences were introduced. Here, we continue this direction of research, introducing two new 3-Fibonacci sequences that differ from the previous two sequences in the number of initial constants in the definition. So, the series of extensions of the nature of the Fibonacci sequence (see, e.g., [2]) is continued.

\section{Main results}

Let everywhere below, $a, b, c$ be arbitrary real numbers.

The first sequence has the form: $\alpha_{0}=2 a, \beta_{0}=2 b, \gamma_{0}=c$ and for each natural number $n \geq 0$ : 


$$
\alpha_{n+1}=\beta_{n}+\gamma_{n}, \quad \beta_{n+1}=\alpha_{n}+\gamma_{n}, \quad \gamma_{n+1}=\frac{\alpha_{n+1}+\beta_{n+1}}{2}+\gamma_{n}
$$

The first members of sequences $\left\{\alpha_{n}\right\}_{n=0}^{\infty},\left\{\beta_{n}\right\}_{n=0}^{\infty}$ and $\left\{\gamma_{n}\right\}_{n=0}^{\infty}$ are the following (Table 1).

\begin{tabular}{|c|c|c|c|}
\hline$n$ & $\alpha_{n}$ & $\gamma_{n}$ & $\beta_{n}$ \\
\hline \hline 0 & $2 a$ & $c$ & $2 b$ \\
0 & & $a+b+2 c$ & $2 a+c$ \\
\hline 1 & $2 b+c$ & & $a+3 b+3 c$ \\
1 & & $3 a+3 b+5 c$ & \\
\hline 2 & $3 a+b+3 c$ & & $6 a+4 b+8 c$ \\
2 & & $8 a+8 b+13 c$ & \\
\hline 3 & $4 a+6 b+8 c$ & & $12 a+14 b+21 c$ \\
3 & & $21 a+21 b+34 c$ & \\
\hline 4 & $14 a+12 b+21 c$ & & $35 a+33 b+55 c$ \\
4 & & $55 a+55 b+89 c$ & \\
\hline 5 & $33 a+35 b+55 c$ & & $88 a+90 b+144 c$ \\
5 & & $\ldots$ & $\ldots$ \\
\hline 6 & $90 a+88 b+144 c$ & & \\
6 & & $\ldots$ & \\
\hline$\ldots$ & $\ldots$ & & \\
\hline
\end{tabular}

Table 1. The first members of the sequences values of sequences

$$
\left\{\alpha_{n}\right\}_{n=0}^{\infty},\left\{\beta_{n}\right\}_{n=0}^{\infty} \text { and }\left\{\gamma_{n}\right\}_{n=0}^{\infty}
$$

Let $\left\{F_{n}\right\}_{n=0}^{\infty}$ be the standard Fibonacci sequence, where $F_{0}=0, F_{1}=1$, and

$$
F_{n+2}=F_{n+1}+F_{n}
$$

for each natural number $n \geq 0$.

Theorem 1. For each natural number $n \geq 1$ :

$$
\begin{aligned}
& \alpha_{n}=\left(F_{2 n-1}+(-1)^{n}\right) a+\left(F_{2 n-1}-(-1)^{n}\right) b+F_{2 n} c \\
& \beta_{n}=\left(F_{2 n-1}-(-1)^{n}\right) a+\left(F_{2 n-1}+(-1)^{n}\right) b+F_{2 n} c, \\
& \gamma_{n}=F_{2 n} a+F_{2 n} b+F_{2 n+1} c .
\end{aligned}
$$

Proof: We can prove the Theorem, for example, by induction. For $n=1$, the validity of the Theorem is checked directly from the above table. Let us assume that the Theorem is valid for some natural number $n \geq 1$. Then:

$$
\begin{aligned}
\alpha_{n+1} & =\beta_{n}+\gamma_{n} \\
& =\left(F_{2 n-1}-(-1)^{n}\right) a+\left(F_{2 n-1}+(-1)^{n}\right) b+F_{2 n} c+F_{2 n} a+F_{2 n} b+F_{2 n+1} c \\
& =\left(F_{2 n+1}-(-1)^{n}\right) a+\left(F_{2 n+1}+(-1)^{n}\right) b+F_{2 n+2} c \\
& =\left(F_{2 n+1}+(-1)^{n+1}\right) a+\left(F_{2 n+1}-(-1)^{n+1}\right) b+F_{2 n+2} c
\end{aligned}
$$




$$
\begin{aligned}
\beta_{n+1} & =\alpha_{n}+\gamma_{n} \\
& =\left(F_{2 n-1}+(-1)^{n}\right) a+\left(F_{2 n-1}-(-1)^{n}\right) b+F_{2 n} c+F_{2 n} a+F_{2 n} b+F_{2 n+1} c \\
& =\left(F_{2 n+1}+(-1)^{n}\right) a+\left(F_{2 n+1}-(-1)^{n}\right) b+F_{2 n+2} c \\
& =\left(F_{2 n+1}-(-1)^{n+1}\right) a+\left(F_{2 n+1}+(-1)^{n+1}\right) b+F_{2 n+2} c \\
\gamma_{n+1}= & \frac{\alpha_{n+1}+\beta n+1}{2}+\gamma_{n} \\
= & \frac{1}{2}\left(\left(F_{2 n+1}+(-1)^{n+1}\right) a+\left(F_{2 n+1}-(-1)^{n+1}\right) b+F_{2 n+2} c\right. \\
+ & \left.\left(F_{2 n+1}-(-1)^{n+1}\right) a+\left(F_{2 n+1}+(-1)^{n+1}\right) b+F_{2 n+2} c\right)+F_{2 n} a+F_{2 n} b+F_{2 n+1} c \\
= & F_{2 n+1} a+F_{2 n+1} b+F_{2 n+2} c+F_{2 n+2} c+F_{2 n} a+F_{2 n} b+F_{2 n+1} c \\
= & F_{2 n+2} a+F_{2 n+2} b+F_{2 n+3} c .
\end{aligned}
$$

The rest formulas are checked by analogy.

The second sequence has the form: $\alpha_{0}=a, \beta_{0}=2 b, \gamma_{0}=2 c$ and for each natural number $n$ :

$$
\alpha_{n+1}=\alpha_{n}+\frac{\beta_{n}+\gamma_{n}}{2}, \quad \beta_{n+1}=\alpha_{n+1}+\gamma_{n}, \quad \gamma_{n+1}=\alpha_{n+1}+\beta_{n}
$$

The first members of the sequences $\left\{\alpha_{n}\right\}_{n=0}^{\infty},\left\{\beta_{n}\right\}_{n=0}^{\infty}$ and $\left\{\gamma_{n}\right\}_{n=0}^{\infty}$ are the following (Table 2).

\begin{tabular}{|c|c|c|c|}
\hline$n$ & $\beta_{n}$ & $\alpha_{n}$ & $\gamma_{n}$ \\
\hline \hline 0 & & $a$ & $2 c$ \\
0 & $2 b$ & $a+b+c$ & \\
\hline 1 & & & $a+3 b+c$ \\
1 & $a+b+3 c$ & $2 a+3 b+3 c$ & \\
\hline 2 & & & $3 a+4 b+6 c$ \\
2 & $3 a+6 b+4 c$ & $5 a+8 b+8 c$ & \\
\hline 3 & & & $8 a+14 b+12 c$ \\
3 & $8 a+12 b+14 c$ & $13 a+21 b+21 c$ & \\
\hline 4 & & & $21 a+33 b+35 c$ \\
4 & $21 a+35 b+33 c$ & & $55 a+90 b+88 c$ \\
\hline 5 & & $89 a+144 b+144 c$ & \\
5 & $55 a+88 b+90 c$ & & $144 a+232 b+234 c$ \\
\hline 6 & & $\ldots$ & $\ldots$ \\
6 & $144 a+234 b+232 c$ & & \\
\hline & $\ldots$ & $\ldots 5+55 c$ & \\
\hline
\end{tabular}

Table 2. The first members of the sequences values of sequences $\left\{\alpha_{n}\right\}_{n=0}^{\infty},\left\{\beta_{n}\right\}_{n=0}^{\infty}$ and $\left\{\gamma_{n}\right\}_{n=0}^{\infty}$ 
Theorem 2. For each natural number $n \geq 1$ :

$$
\begin{aligned}
& \alpha_{n}=F_{2 n-1} a+F_{2 n} b+F_{2 n} c, \\
& \beta_{n}=F_{2 n} a+\left(F_{2 n+1}+(-1)^{n}\right) b+\left(F_{2 n+1}-(-1)^{n}\right) c, \\
& \gamma_{n}=F_{2 n} a+\left(F_{2 n+1}-(-1)^{n}\right) b+\left(F_{2 n+1}+(-1)^{n}\right) c .
\end{aligned}
$$

Other new schemes, modifying the standard form of the 2- and 3-Fibonacci sequences and the above two sequences, will be discussed in near future.

\section{References}

[1] Atanassov, K. (2018) On two new combined 3-Fibonacci sequences. Notes on Number Theory and Discrete Mathematics, 24(2), 90-93.

[2] Atanassov, K., Atanassova, V., Shannon, A., \& Turner, J. (2002) New Visual Perspectives on Fibonacci Numbers World Scientific, New Jersey. 